\title{
ENSEÑANZA POR CAMBIO CONCEPTUAL: DE LA FÍSICA CLÁSICA A LA RELATIVIDAD
}

\author{
ALEMAÑ BERENGUER, RAFAEL ANDRÉS ${ }^{1}$ y PÉREZ SELLES, J.F. ${ }^{2}$ \\ ${ }^{1}$ IES Pere M. Orts i Bosch. Benidorm. Alicante \\ ${ }^{2}$ IES núm. 4. Benidorm. Alicante
}

\begin{abstract}
SUMMARY
In this article the possibility of learning through conceptual change and the best way to achieve this aim, are discussed. This teaching needs to have certain specific features and ensure that the debate in the classroom is focused on the explicit ideas of the students, whose conceptual difficulties must be taken into account with considerable care. To obtain those results, we are to give critical importance to the explanation and justification of ideas, debates must be metacognitive and professors should share discussion in an active and diversified role. One example of this way of teaching is introduced in this article concerning the conceptual change required to pass from classical to relativistic physics.
\end{abstract}

\section{INTRODUCCIÓN}

Nadie cuestiona hoy día el papel básico desempeñado por el conocimiento real de los estudiantes en cualquier actividad de aprendizaje intelectual. Este conocimiento real, mezcla de los contenidos aprendidos y de las ideas preconcebidas, muestra una considerable diversidad entre los estudiantes, resulta a menudo contradictorio consigo mismo y diverge con frecuencia de la instrucción recibida. Todo ello indica que el genuino proceso de aprendizaje en el ser humano procede, no por acumulación simple de conocimientos, sino por sustitución de los conceptos antiguos por los nuevos, en el caso de la transición de la física del sentido común a la física newtoniana, o por subsunción de unos esquemas conceptuales bajo otros, como en el paso de la física clásica a la relatividad. Esta actuación ha sido denominada por los psicopedagogos como aprendizaje por cambio conceptual (Hewson, 1981, 1982; Posner et al., 1982; Hewson-Thorley, 1989; Thorley, 1990; Strike-Posner, 1985, 1992), mientras que los psicólogos cognitivos han des- tacado la importancia de que las ideas aprendidas formen «cadena de significantes» con el resto del conocimiento asimilado (Vygotsky, 1978; Evart et al., 1984; Marler y Terrace, 1984). Esto significa, ni más ni menos, que los nuevos conceptos han de modificar los contenidos previamente aceptados como válidos, a la vez que se asumen todas sus posibles implicaciones lógicas y epistemológicas tanto consciente como inconscientemente. En esta vía para alcanzar un aprendizaje verdaderamente significativo, el cambio no sólo ha de darse en el nivel conceptual sino también en el de la metodología (Gil y Carrascosa, 1985) y las actitudes.

Tal concepción constructivista del aprendizaje implica la consideración de dos cuestiones relacionadas: el significado del aprendizaje como cambio conceptual llevado a cabo por los propios estudiantes bajo la supervisión del docente y la enseñanza como un proceso de encadenamiento de sucesivos cambios conceptuales a medida 
que el alumno progresa en su desarrollo intelectual y cognitivo (Laburu, 1996). En cuanto a la primera cuestión, es imprescindible tomar en cuenta la influencia del marco conceptual previo del discente. En él se sitúan tanto la valoración de las ideas que sostiene, en función de su inteligibilidad, verosimilitud y utilidad, como el resto de sus ideas previas. La importancia de estas últimas resulta difícil de exagerar, pues es a través de ellas, a modo de anteojos psicológicos, como el estudiante analiza los conocimientos adquiridos, y en ellas se ubica la principal fuente de resistencia al cambio conceptual efectivo (Scott et al., 1992). A partir de ello se realiza el segundo paso, en el cual el aprendizaje se produce mediante una sucesión de cambios conceptuales encadenados (Hewson y Beeth, 1995) que pueden darse, o bien por desplazamiento de unos paradigmas por otros (paso de la errónea física aristotélica a la newtoniana), o bien por inclusión de los precedentes en el interior de los nuevos como casos particulares de estos últimos (transición de la física de Newton a la de Einstein).

En todas las discusiones al respecto, se destacan algunos aspectos relevantes. Uno de ellos es la citada influencia de los conocimientos previos -en ocasiones juzgados como un obstáculo a superar y en otras contemplados como la oportunidad de base para un nuevo aprendizajejunto al papel del profesor y las correspondientes controversias sobre la amplitud del control que éste ha de ejercer sobre el aprendizaje de los alumnos. De un modo u otro, en este artículo se esbozarán las normas esenciales de la enseñanza por cambio conceptual, considerando varios factores relevantes a este respecto (profesor, estudiantes y clima del aula). A continuación se aplicarán tales apreciaciones a un ejemplo referido al cambio conceptual implicado en la transición desde el pensamiento físico clásico al de la relatividad einsteniana, por lo cual, más que orientarse a la mera aplicación de un marco didáctico determinado (el constructivista), el presente trabajo debe entenderse como la contractación de su utilidad en el caso concreto de la enseñanza de temas sobre relatividad.

\section{ORIENTACIONES SOBRE LA ENSEÑANZA POR CAMBIO CONCEPTUAL}

Las ideas expuestas en lo que sigue deben tomarse como guías metodológicas flexibles y ampliamente adaptables a las necesidades específicas de cada situación concreta de enseñanza-aprendizaje. Asimismo, el hecho de presentarlas secuencialmente no significa orden de prelación alguno, puesto que cada una de las pautas siguientes presentan diferentes finalidades susceptibles de ser alcanzadas simultáneamente a partir de la actividad elegida en el aula.

\section{Debate explícito de las ideas previas y adquiridas de los estudiantes}

En este género de enseñanza, es de crítica importancia que las ideas previas de los alumnos se hagan explícitas durante el transcurso del aprendizaje, del mismo modo que así debe hacerse con las que vayan adquiriendo en este mismo proceso. Ése es el método más seguro y eficaz para superar los obstáculos levantados por prejuicios inconscientes y para asentar los conocimientos asimilados poniendo de relieve las múltiples acomodaciones y ajustes mutuos que exige a las ideas antiguas la incorporación de nuevos conocimientos.

Esta actitud muestra dos ventajas respecto a la posición tradicional de la relación profesor-alumno. La primera es que el estudiante aprende a conocer y valorar las opiniones de sus compañeros tanto como las del profesor o las suyas propias, de manera que se habitúa a contemplar con naturalidad la posibilidad de discrepar sobre una cuestión que sólo debe quedar concluida tras un debate razonado. La segunda ventaja consiste en que el estudiante aprende a decidir sobre la aceptación o rechazo de las ideas que se le proponen, no sobre la base de la autoridad, sino mediante el raciocinio (Osborne y Freyberg, 1985). En lugar de limitarse a aceptar y repetir pasivamente las ideas y concepciones transmitidas por el profesor, un debate en el curso del cual se plantea el cuestionamiento de las opiniones de todos los presentes demuestra ser el mejor camino para la obtención de una verdadera asimilación de los conceptos en un nivel consciente tanto como en el subconsciente. Como es lógico, puede llegarse a un punto -y frecuentemente se llega- en el que el alumno ha de aceptar los hechos y teorías elaborados desde largo tiempo atrás por el conjunto de la comunidad científica mundial. Pero, en todo caso, el examen objetivo de los conocimientos antes de ser asumidos por el estudiante le dota de un hábito de pensamiento crítico que facilitará la aceptación de aquellos contenidos que simplemente deba admitir, en la confianza de que futuros procesos de enseñanza-aprendizaje le permitirán considerarlos inteligibles en la misma medida que los anteriores.

Algunas muestras de metodología didáctica destinada a la elucidación de los preconceptos de los alumnos son las siguientes:

1) El profesor comienza con un cuestionario individual (que no comporte calificaciones) confeccionado con preguntas que presenten un cierto margen de opciones relacionadas con diversas opiniones corrientes. Tras el cuestionario, el profesor describe la muestra de las respuestas dadas en clase y pide a los estudiantes que expliquen sus opiniones (Minstrell, 1982).

2)Los estudiantes definen lo que significa para ellos un término o concepto antes de la clase lectiva (Hennessey, 1991) y reelaboran dicho significado en función del aprendizaje subsiguiente.

3) El profesor ofrece algunos ejemplos para ilustrar el contexto de trabajo y los alumnos abordan la cuestión mediante actividades individuales cuyos resultados discuten por parejas y más tarde presentan al conjunto de la clase (Clis, 1987). 
En el presente artículo optaremos por un modelo ecléctico. Dadas las sutilezas de la materia elegida (paso de la física clásica a la relativista), la introducción al tema -pues no se excederá de un nivel introductorio-se realizará de forma guiada por el profesor. Pero al mismo tiempo las dificultades surgidas en el curso de este proceso se aclararán a medida que se hagan explícitas reconsiderando el significado de los términos y la extensión de los conceptos propiciadores de la confusión.

\section{Valoración y análisis crítico de las ideas puestas en discusión}

La enseñanza por cambio conceptual induce en los alumnos un proceso de supervisión y valoración crítica de sus propias ideas. En el curso de las discusiones que constituyen el proceso de aprendizaje desde esta perspectiva pedagógica, el estudiante se ve en la necesidad de considerar conceptos y opiniones diferentes a los suyos propios, eligiendo entre unos u otros de acuerdo con la información disponible y con su adecuación al problema de que se trate. Rechazar sus ideas previas y sustituirlas por otras que ahora crea más adecuadas, cambiar el valor relativo de tales ideas previas dentro de un marco conceptual e ideológico modificado por la adquisición de nuevos conocimientos o, incluso, permanecer con las ideas propias pero tras haberlas aquilatado y aclarado son algunas de las opciones a que nuestro procedimiento da lugar.

La mayor parte de las veces, el alumno prefiere las explicaciones capaces de proporcionarle los mecanismos causales que le resulten más familiares. En esos casos nos enfrentamos al problema de persuadirles que no siempre los procesos causales a los que estamos habituados son los que realmente operan en la naturaleza, especialmente si nos referimos a fenómenos y ámbitos muy alejados de nuestra experiencia cotidiana. Debemos recurrir, entonces, a ciertas estrategias lógicas, como la de exigir explicaciones similares en situaciones similares, proporcionar ejemplos desacostumbrados, aplicar las mismas ideas en contextos diversos, ofrecer distintas maneras de enfocar las cuestiones para enlazar con otras ideas, etc.

Todas estas técnicas didácticas tendrán tanto más éxito cuanto más capte el estudiante la imperfección de la idea que el aprendizaje por cambio conceptual intenta reemplazarle. Un obstáculo común reside en la frecuente y errónea suposición de los profesores de que la discrepancia a partir de la cual pretenden llevar a cabo el cambio conceptual resulta tan obvia para el estudiante como para ellos mismos. Muy a menudo no resulta así y el alumno se ve forzado a admitir sin demasiada convicción los nuevos contenidos. El resultado final -como todos sabemos- es que los conceptos previos no desaparecen por sustitución sino que persisten como un sustrato, muchas veces indeseable, por debajo de los nuevos aprendizajes realizados.

\section{Justificación explícita de las ideas discutidas, acepta- das o rechazadas}

Con el fin de llevar a la práctica un modelo de enseñanza por cambio conceptual, resulta indispensable que los alumnos determinen por sí mismos -aunque con ayuda del profesor, naturalmente- el grado de validez que una idea tiene para ellos. Para ese propósito, primero han de ser conscientes de la categoría de esa idea; es decir, saber si es comprensible para ellos y decidir después si encuentran esa idea verosímil o, al menos, instrumentalmente útil. La comprensibilidad de una idea suele expresarse a través de la capacidad del alumno para interpretar el sentido de las palabras y frases empleadas en formularla, así como la posibilidad de hablar sobre ella explicándola a terceros o buscando analogías y ejemplos que la ilustren.

La verosimilitud de una idea, en cambio, se adquiere a juicio del estudiante, o bien porque se crea que es cierta, o bien por considerarla consistente con otros conocimientos tenidos por ciertos o plausibles. Uno de los dos criterios principales de verosimilitud es el metafísico, el referido a las creencias que no pueden probarse porque atañen a la naturaleza misma de las cosas; el otro es el criterio epistemológico, utilizado por una persona para decidir si un conocimiento se halla justificado o no. El criterio epistemológico se funda generalmente en la consistencia lógica mutua: si alguien cree que una idea es significativamente consistente con otra idea aceptable, ello se convierte en base suficiente para decidir que también la primera lo es. La posibilidad de imaginar mecanismos causales creíbles, desempeña, por supuesto, un importante papel en la verosimilitud otorgada a una idea.

\section{Otros factores de importancia en la enseñanza por cambio conceptual}

En esta opción didáctica, la función del profesor, lejos del mero transmisor repetitivo de conocimientos, cobra una especial relevancia. Por un lado, ha de jugar el papel de guía capaz de indicar los caminos más directos y eficaces para lograr el objetivo deseado -el aprendizaje significativo- planteando problemas instructivos, explorando las distintas ideas con respeto a todos sus proponentes y estableciendo tareas de refuerzo en las que los alumnos practiquen los conocimientos recién adquiridos. Por otro lado, ha de ser también un participante activo en la clase, mostrando a los estudiantes, con su ejecutoria, la fertilidad de la enseñanza por descubrimiento y dejando bien claro que la experiencia y el razonamiento lógico -por oposición a la autoridad y la tradición-son las únicas fuentes de fiabilidad en la ciencia.

Los alumnos deben aceptar la responsabilidad de su propio aprendizaje, hacerse conscientes de sus mismas ideas y de sus propias razones para aceptarlas, confiar en su propio pensamiento, y justificar sus conclusiones utilizando argumentos sensatos. Estas ambiciosas metas pedagógicas se logran con mucha mayor facilidad si se 
logra crear el clima adecuado en el aula. Así, es imprescindible respetar las ideas ajenas y prestarles atención aun cuando no se esté de acuerdo con ellas. El paso inmediatamente posterior es el de lograr la facultad de criticar racionalmente una idea al mismo tiempo que se acepta a la persona que la aporta. En todo ello ha de realizarse un esfuerzo por entender los puntos de vista de los demás (tanto profesores como alumnos), adoptando las conclusiones finales, no porque alguien lo diga, sino porque tengan significado para los participantes en la discusión.

Una característica del proceso de cambio conceptual, a la vez causa y efecto del aprendizaje significativo, es la puesta en práctica de un debate metacognitivo, entendido como la revisión del «conocimiento acerca de los procesos y resultados cognitivos de uno mismo» (Flavell, 1976). Cuando el profesor presenta diversas explicaciones para un mismo fenómeno fomentando la controversia sobre las mismas, o cuando los alumnos comentan, comparan y contrastan, considerando los argumentos a favor y en contra, se encuentran realizando actividades metacognitivas relacionadas con las exigencias de la tarea de aprendizaje y las estrategias utilizadas para aprender (Palincsar y Ransom, 1988).

Hemos de insistir, por último, en una idea ya comentada brevemente en la introducción. A diferencia de lo que ocurre cuando se aplica la didáctica de las ciencias a la enseñanza de los conceptos básicos de la física, en este caso no cabe afirmar que partimos de un paradigma conceptual abierta o necesariamente falso. En efecto, de donde se parte es del conjunto de ideas de la física newtoniana, las cuales han sido asumidas por los alumnos como extraordinariamente adecuadas y exitosas en la explicación de la naturaleza, obtenidas a lo largo de los cursos precedentes. Ya no se trata, pues, de una mera sustitución de conocimiento «vulgar» por conocimiento «científico», sino más bien de la presentación de una teoría más potente y abarcadora capaz de incluir en su seno las teorías antecesoras. El aprendizaje por los alumnos de los rudimentos de la mecánica relativista, desde luego, no debe conllevar el menosprecio de la mecánica newtoniana tan fructífera y útil en numerosos problemas cotidianos y científicos. En esta línea, por tanto, los alumnos deben concebir el aprendizaje de nuevas teorías científicas como el enriquecimiento de un abanico de opciones entre modelos alternativos cuyo poder explicativo resulta ser progresivamente creciente, aunque sin desechar por erróneo ninguno de ellos (Mosterín, 1987).

\section{EJEMPLO DE ENSEÑANZA POR CAMBIO CONCEPTUAL: INTRODUCCION A LA RE- LATIVIDAD}

Vamos a ilustrar a continuación las pautas reseñadas hasta ahora con un ejemplo práctico vinculado a la transición que se produce en el marco conceptual de los alumnos al acceder a la física relativista partiendo de la clásica. La problemática a tratar en el curso de estas sesiones será la confrontación entre los conceptos de espacio y tiempo desde la perspectiva de ambas físicas (clásica y relativista), pues es lo que creímos más adecuado para introducir en los alumnos los rasgos elementales de la teoría de la relatividad especial. Los puntos tratados serán:

- Simultaneidad de sucesos

- Dilatación de duraciones y contracción de longitudes

- El tiempo como cuarta dimensión

- El espacio-tiempo curvo de la relatividad general y la expansión del universo

Además de su importancia en el marco de la teoría, estos apartados fueron seleccionados sobre la base del interés mostrado por los propios alumnos en el curso de conversaciones exploratorias previas. Lo exóticas y excitantes que parecen estas ideas, así como la cantidad de confusiones propiciadas por el cine y la literatura de cienciaficción, favorecerán a buen seguro la discusión.

\section{Metodología de la investigación}

El caso estudiado se centra en un grupo de seis estudiantes que libremente ( $\sin$ presiones o sugerencias de calificaciones positivas o negativas) decidieron recibir instrucción suplementaria sobre este tema en el transcurso de un seminario voluntario realizado al final del año lectivo 1997-98 (abril-mayo de 1998). Los alumnos ( 4 chicos y 2 chicas) pertenecían al $2^{\circ}$ curso de bachillerato LOGSE y todos ellos presentaban las características típicas de los alumnos de su nivel de desarrollo intelectual y emocional, sin que ninguno precisase de refuerzos docentes ni ayudas especiales de tipo alguno.

Durante los cursos anteriores y hasta el momento de la experiencia, habían mostrado un dominio correcto de la mecánica clásica (leyes de Newton, relatividad de Galileo), y todos ellos justificaron su participación por la atracción y el interés que les despertaba el tema a tratar en el seminario. Las reuniones se realizaron en el seminario de física y química, lugar con el que dichos alumnos se hallaban familiarizados por el desarrollo de las clases de otras asignaturas optativas. Las sesiones se extendieron durante cuatro tardes, a razón de una sesión de tres horas a la semana, distribuidas en dos períodos de ochenta minutos con un descanso intermedio de veinte. En cada una de dichas sesiones se pasó revista mediante la técnica del debate dirigido a los principales puntos de la teoría de la relatividad, confrontando los nuevos puntos de vista con los adquiridos por los estudiantes a lo largo de su instrucción previa. Las sesiones, si no enteramente, sí en los tramos juzgados de mayor interés, fueron grabadas magnetofónicamente con el consentimiento general y bajo el compromiso explícito de los docentes de respetar el anonimato de los alumnos. Reproduciremos más adelante algunas de tales intervenciones, en la que los nombres de los participantes han sido sustituidos por las letras del alfabeto (naturalmente, 
cuando en otros apartados vuelvan a transcribirse fragmentos del debate, no siempre las mismas letras corresponderán a los mismos intervinientes).

\section{Determinación de la simultaneidad de dos sucesos}

Las sesiones comienzan discutiendo la idea de simultaneidad entre acontecimientos separados espacialmente. Para ello se utiliza el ejemplo que muestra las diferentes opiniones de dos observadores sobre el momento de caída de dos rayos. Cuando el primero se encuentra parado en tierra, el segundo está subido a un tren que se mueve, de modo que los rayos están acercándose a uno de ellos y alejándose del otro. Inicialmente la posición de los dos observadores es la misma en relación con el camino que debe seguir la luz hasta ambos. En concreto, estos dos observadores se encuentran a la mitad de ese recorrido en el instante inicial. Mediante sencillos dibujos, los alumnos no tienen dificultades en deducir que, si el observador terrestre juzga simultánea la caída de ambos rayos, el del tren considerará que ocurre antes la caída del rayo hacia el que él mismo se aproxima.

$A$ : «Está claro que si, para el observador del suelo, los dos rayos caen a la vez, es porque su reloj marca la misma hora cuando la luz de los dos le llega a él. Pero eso no puede pasarle al del tren.»

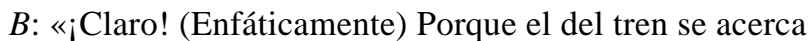
a uno de los rayos mientras se aleja del otro. Por eso la luz de uno le llegará antes que la del otro, y para él uno habrá ocurrido antes que el otro.»

$A$ : «Ésa es la razón por la que los dos observadores no se ponen de acuerdo en la simultanedidad de los sucesos.»

$C:$ «Pero eso es un efecto del movimiento del tren. Igual que cuando voy en un tren y me parece que se mueven los árboles al lado de la vía. Pero yo sé que de verdad no se mueven, que es una apariencia debida a mi velocidad.»

$B$ : «Sí, y por eso es el observador del suelo el que tiene razón porque se encuentra en reposo y ningún movimiento lo perturba.»

No obstante, se detecta una tendencia espontánea a creer que la percepción del observador en tierra es, en cierto modo, «más real» que la del viajero del tren. Al fin y al cabo -se dice-, «el observador terrestre se encuentra en reposo». Es preciso recodarles en ese momento que el principio de relatividad exige que los sistemas de referencia considerados en reposo y los considerados en movimiento inercial resulten físicamente equivalentes. Un esfuerzo consciente por aplicar en esta situación las exigencias de este principio nos lleva a concluir finalmente que la idea de simultaneidad es relativa al referencial en que se determine. Como se ha visto, los alumnos se muestran resistentes a aplicar el principio de relatividad en aquellos casos en que su concepcion del tiempo absoluto - heredada, sin duda, de la física newtonianase vea puesta en entredicho. Pese a todo, este primer peldaño de cambio conceptual ha sido incompleto, como se verá en el apartado siguiente.

\section{Dilatación temporal y contracción de longitudes}

La conjunción del principio de relatividad con la constancia de la velocidad de la luz (c) nos permite deducir efectos peculiares de la teoría de Einstein, como la asignación de distintas duraciones para un mismo proceso dependiendo del sistema de referencia con respecto al cual se determine dicha duración. Fue muy interesante el debate mantenido por los estudiantes entre sí acerca de la interpretación de este punto.

$A$ : «Supongo que será de un problema de medida. Al moverse a velocidades próximas a la de la luz, los observadores no pueden ajustar sus mediciones y así les parece que los intervalos de tiempo salen mayores.»

$B$ : «No creo que sea eso, porque, si fuese un problema de medidas, tanto podrían salir duraciones mayores como menores -entre los sistemas de referencia en movimiento relativo, quiero decir-. Y no sería como dicen las fórmulas, una "dilatación de tiempos".»

$A$ : «¿Pero por qué no puede ocurrir ese desajuste de las medidas de manera que sea siempre una dilatación del tiempo? Nadie puede saber "porque sî" lo que pasa a la velocidad de la luz.»

En este momento interviene el profesor para puntualizar que un error muy extendido es el del operacionalismo, que atribuye todo significado físico a las operaciones de medida. Por el contrario, las ecuaciones de la física ni mencionan observadores ni aparatos de medida, sino que conciernen a los fenómenos naturales en sí mismos. De hecho, las teorías de la medida son herramientas científicas importantes, complementarias de las teorías fundamentales y necesarias para su verificación, pero incapaces de sustituirlas.

$C$ : «Tal vez ocurra que los movimientos del observador que se mueve se hacen más lentos, como cuando baja la temperatura, y es por eso que las duraciones se agrandan.»

$A$ : «Pero, si fuese así, no se explica por qué es un efecto mutuo. Además, cuando las cosas van más despacio al bajar la temperatura, nosotros podemos saber dónde la temperatura ha bajado y dónde no, pero la relatividad dice que ambos sistemas son equivalentes.»

$D:$ «A mí me parece que debe ser algo así como un efecto óptico, igual que cuando me alejo de un amigo y después de andar mucho terreno lo veo muy lejos y muy pequeño, y él me ve a mí igual aunque los dos sabemos que ninguno ha empequeñecido.»

$C:$ «Pero eso es parecido a lo del espejismo. La dilatación del tiempo no es una cosa óptica porque no depende sólo de nuestros ojos, y es que se manifiesta en muchos fenómenos más.» 
Tras algunos minutos más de discusión, el profesor aclara que la dilatación de las duraciones es un efecto que atañe a la misma naturaleza del tiempo y no puede atribuirse a medidas defectuosas o deceleración de movimientos. Es necesario otro esfuerzo intelectual consciente para rechazar los patrones de tiempo absoluto adquiridos en la física newtoniana, la cual incorpora a su vez presunciones fuertemente arraigadas en el sentido común.

La contracción de las longitudes recibe un planteamiento similar al del tiempo, y ahora los estudiantes se encuentran más receptivos ante opciones antes difícilmente imaginables por ellos. De todos modos, se ensayan primero las hipótesis más «razonables»-así lo dicen- referidas a posibles efectos ópticos que hagan aparecer los objetos contraídos o las deformaciones elásticas producidas por alteraciones en la estructura molecular ocasionadas por la velocidad. Finalmente se concluye que las longitudes, como las duraciones, no son propiedades absolutas de las cosas, sino dependientes del sistema de referencia. Por ello se parecen más a relaciones del tipo «estar cerca de...» al no ser independientes del marco de referencia en que se determinan.

$A$ : «A mí me parece increíble saber que a distintas velocidades el tiempo corre de distinta manera.»

$B$ : «Pues a mí eso me parece menos difícil de creer que lo de la variación de las longitudes... Vamos, no es que las longitudes varíen, sino que el concepto de longitud depende del movimiento del sistema de referencia. Y es que, mientras que yo el tiempo no lo veo ni lo toco, y si me dicen que en unos sitios pasa más deprisa que en otros, me lo puedo creer, ique me digan que esta mesa no es lo grande que yo veo que es!»

Una mención a la demostración experimental de estos efectos, en experiencias con partículas llamadas muones (French, 1991), refuerza la confianza de los alumnos en la veracidad de los resultados.

\section{El tiempo y la cuarta dimensión}

Los alumnos admiten previamente haber oído hablar del tiempo como de la cuarta dimensión, pero en otros contextos (generalmente de ciencia-ficción) esa cuarta dimensión se les presenta como una especie de mundo invisible que existe junto a nosotros y por el que quizás se podría transitar. Explicarles que el espacio y el tiempo se combinan en un entramado de cuatro dimensiones denominado espacio-tiempo, no disipa todas las dudas. Si el tiempo es una suerte de cuarta dimensión -se preguntan-, ¿por qué no podemos viajar adelante y atrás en ellas como lo hacemos en las tres direcciones espaciales ordinarias? La respuesta parece obvia cuando los estudiantes se liberan de sus hábitos mentales corrientes: porque el tiempo no es la cuarta dimensión del espacio, sino de un concepto distinto, como es el espacio-tiempo. Por eso no se le pueden asignar las mismas propiedades que a las dimensiones espaciales. Una analogía propuesta por el profesor ayuda a intuir algo del particular: el conjunto de las vigas de un edificio forma una estructura única, pero no es lo mismo intentar caminar por el suelo que por la dirección perpendicular de las paredes.

El hecho de que el espacio-tiempo sea un concepto distinto al del espacio y el tiempo por separado permite introducir una nueva fórmula para calcular la «distancia» entre dos puntos del espacio-tiempo (en cierta analogía con los puntos del espacio). Así:

$(\text { Separación espacio-temporal })^{2}=($ Separación temporal $)^{2}-(\text { Separación espacial })^{2}$

Esta nueva fórmula permite explicar la razón de la famosa «paradoja de los gemelos», puesto que ahora el gemelo que viaja y regresa recorre una distancia espacio-temporal mayor que el que permanece en la Tierra (Resnick, 1981; Alemañ, 1998).

Una consecuencia de esta idea de la realidad física que invariablemente levanta grandes resistencias por parte de los alumnos es la que afirma que pasado, presente y futuro permanecen tan fijos en ese espacio-tiempo de cuatro dimensiones como la distancia MadridBarcelona en el espacio tridimensional ordinario. Al introducir el tiempo como una coordenada más, se elimina cualquier posibilidad de considerar el «flujo» del tiempo más que como una ilusión psicológica (Einstein, 1985). Los estudiantes se oponen con vehemencia a esta noción arguyendo que la propia trama del espaciotiempo puede estar desplegándose, de modo que sólo el pasado persistiría desde una perspectiva cuadridimensional.

$A$ : «Parece que nos están diciendo que mi pasado, mi presente y mi futuro están ya puestos en el espaciotiempo, como si fueran una película de cine que se está proyectando, pero cuyo final está ya contendido en el rollo de la cinta.»

$B$ : «Bueno, ¿pero a qué velocidad se proyecta esa «película»? ¿No habíamos quedado que el tiempo pasa a distinto ritmo según el sistema de referencia? ¿O es que para algunos observadores ya me han pasado cosas que todavía no me han pasado?»

$C$ : «(En tono humorístico) A lo mejor sí te han pasado pero aún no te has dado cuenta.»

La inconsistencia de este argumento se muestra explicando que ese proceso de despliegue se realizaría a un cierto ritmo o velocidad para calcular la cual deberíamos introducir una nueva coordenada de tiempo -ya que el tiempo ordinario se halla incluido en el espaciotiempo-, lo que no adelantaría nada, pues siempre podríamos insertarla en un espacio-tiempo con más dimensiones. Con este razonamiento, si no completamente convencidos, los alumnos son, por lo menos, conscientes de la insuficiencia de su imagen de la naturaleza como una realidad tridimensional cuyo continuo fluir en el tiempo es puramente aparente. 


\section{Relatividad general. Espacio-tiempo curvo}

Avanzando un paso más en nuestra excursión -nunca excediendo un nivel puramente cualitativo- por la relatividad, se plantea a los participantes en el seminario la inconsistencia de la gravitación newtoniana con la teoría de Einstein, pues Newton daba por supuesta la transmisión instantánea de la atracción gravitacional, mientras que la relatividad prohíbe la propagación de señales físicas más rápidas que la luz. Los alumnos aceptan y comprenden esta dificultad, con lo que a continuación se propone la solución einsteniana expresada en su teoría de la relatividad general. La gravedad es en realidad un efecto de la curvatura del espacio-tiempo ocasionada por la presencia de masas en su seno, al igual que una lámina elástica se curva al depositar un peso sobre ella.

En este punto nos topamos con dos tipos de escollos intelectuales: el primero se debe a la incapacidad de los estudiantes para imaginarse la curvatura del espacio, ya que «si el espacio es la nada, ¿cómo puede curvarse la nada?».

$A$ : «A mí no me cabe en la cabeza que el espacio pueda curvarse o estar curvado, porque, si el espacio es la nada, ¿cómo puede curvarse la nada?»

$B$ : «Sí, no creo que se pueda decir que el espacio sea plano ni curvo, igual que no se puede decir que es espacio «es feliz»o «está triste». La felicidad no es una propiedad del espacio, y la curvatura tampoco, sino de las cosas que se pueden tocar y doblar.»

Es preciso insistir en que el espacio y el tiempo no son como los suponía Newton, una especie de escenarios inamovibles en los que transcurren los acontecimientos del mundo físico. Desde la perspectiva relativista, son entes dinámicos que influyen y son influidos por la distribución de materia y energía en su interior. El término curvatura concierne a ciertas propiedades del espacio-tiempo que se manifiestan por el comportamiento de la materia en su interior, y no porque éste sea como un objeto tangible cuya torsión cabe observar «desde fuera».

El segundo obstáculo surge de la costumbre de pensar sólo en la curvatura del espacio en lugar de la del espacio-tiempo. Por ello se extraen conclusiones equivocadas como que «la curvatura del espacio producida por el Sol se manifiesta en las trayectorias curvadas de los planetas en torno a él». El profesor interviene aquí para recordar que, si el espacio estuviese curvado en torno a la Tierra, obligándola a moverse en su órbita, también obligaría a permanecer en sus inmediaciones a todos los satélites artificiales lanzados desde ella. Es la curvatura espacio-temporal (en el espacio-tiempo la trayectoria de la Tierra es semejante a una hélice enroscada) la responsable del movimiento de los planetas, y no una especie de cáscara espacial invisible que los mantiene en sus posiciones respectivas.

\section{La expansión del universo}

Dado que la gravedad es la fuerza que domina a gran escala en la estructura del cosmos, se hace inevitable preguntar por la influencia de estas teorías en nuestra concepción del universo. La primera importante repercusión es la de que resulta posible construir modelos cosmológicos en los que es posible evadir la pregunta sobre los límites del universo: el cosmos es como la superficie de una esfera, no tiene bordes ni fronteras, pero a la vez su tamaño es finito en todo instante. Los estudiantes comprenden rápidamente las dificultades a las que conduce un universo espacialmente infinito, pero no acaban de asimilar la solución ofrecida por la relatividad (Singh, 1982).

$A$ : «Si el universo es como la superficie de una esfera, ¿qué es lo que hay dentro y fuera? Si la esfera se curva en el espacio, ¿dentro de qué se curva el universo?»

$B$ : «La verdad es que yo no puedo imaginarme una esfera sin pensar en el espacio de dentro y el de fuera.»

$C:$ «Sí, intentarlo es como imaginarse un triángulo sin tres lados o sin tres ángulos. Eso no puede ser.»

$D:$ :En un globo que se hincha, el aire de dentro es el que lo hace inflarse. Pero si el universo es como una esfera sin interior ni exterior, ¿qué es lo que causa la expansión?, ¿qué es lo que empuja la superficie de esa esfera cósmica?»

Estos dos comentarios ponen el dedo en la llaga de la dificultad de los alumnos. La costumbre de visualizar en imágenes muchos de los conceptos con que piensan les bloquea cuando llegan a un grado de abstracción en el que esto no es posible. Es necesario, entonces, recordar que los modelos se asemejan a la realidad tan sólo en parte, por lo que no cabe esperar que todas las características de una superficie esférica sean compartidas por el universo en su conjunto. Bastaría con pensar que un viajero que se desplazase continuamente en línea recta en un cosmos como ése volvería al cabo de un tiempo a su punto de partida sin haber virado su rumbo en ningún momento.

La expansión del universo (asunto del que los alumnos ya saben por periódicos, libros y documentales televisivos) se explica mucho mejor en este modelo que con cualquier otro. Puesto que la distancia entre las galaxias crece en proporción directa a la distancia que las separa -lo que es un dato experimental que pocos pondrían hoy en duda-, el punto de vista newtoniano nos obligaría a suponer que la materia del universo se expande a partir de un punto central desplazándose sobre un espacio vacío preexistente. Pero no es eso lo que ocurre, porque no es que las galaxias se desplacen dentro del espacio como los peces se mueven dentro del agua; es más bien que el propio espacio se expande aumentando la separación entre las galaxias sin que éstas se muevan sobre un fondo espacial fijo. El símil de la esfera nos ayuda de nuevo si imaginamos que se trata de una globo inflándose con unas cuantas monedas (las galaxias) pegadas a su 
superficie. No se mueven las monedas sino que el globo se infla separándolas (Reeves, 1996).

\section{CONCLUSIONES}

Dado el alto grado de abstracción de los conceptos manejados en esta experiencia didáctica, no ha sido posible efectuar una enseñanza por descubrimiento del alumno, pero no dudamos que la intervención puntual del profesor para introducir los conceptos difíciles, o animar el debate, bien ha valido la pena. De todo ello pueden extraerse las siguientes conclusiones:

1) Las dificultades que encuentran los alumnos para asimilar el punto de vista de la teoría de la relatividad se deben generalmente a su falta de hábito en concebir el mundo físico como un entramado espacio-temporal de cuatro dimensiones (distinto al simple espacio tridimensional) con unas propiedades geométricas peculiares.

2) Sabido esto, no resulta en exceso complicado plantearles -siquiera en un nivel meramente introductoriolas ideas fundamentales de la relatividad en contraste con las de la física clásica.

3) Los modelos de pensamiento de la física de Newton, cuando son asumidos plenamente por el estudiante, se

\section{REFERENCIAS BIBLIOGRÁFICAS}

ALEMAÑ, R. (1998). Grandes metáforas de la física. Madrid: Celeste ediciones.

CLIS, H. (1987). Approaches to teaching the particulate theory of matter. Leeds: Univ. of Leeds Press.

EINSTEIN, A. (1985). El significado de la relatividad. Barcelona: Planeta.

EVARTS, E.V., SHINODA, Y. y WISE, S.P. (1984) Neuropsychological approaches to higher brain functions. Nueva York: Wiley.

FLAVELL, J.H. (1976). Metacognitive aspects of problem solving, en Resnik, L.B. (ed.). The Nature of Intelligence, pp. 231-235. Nueva Jersey: Hillsdale, Lawrence Erlbaum.

FRENCH, A.P. (1991). Relatividad especial. Barcelona: Reverté.

GIL, D. y CARRASCOSA, J. (1985). Science learning as a conceptual and methodological change. European Journal of Science Education, 7(3), pp. 231-236. resisten a su remodelación con el mismo vigor con que se resistían las ideas de la física ingenua del sentido común (llamada por algunos «física aristotélica») a ser sustituidas por las de Galileo-Newton. Esto indica que, si bien puede hablarse de lograr un cierto aprendizaje conceptual (se reconoce la existencia de nuevos conceptos, distintos a los previamente aprendidos) y procedimental (se discuten algunos de sus formalismos matemáticos aplicados a la resolución de problemas sencillos), en otros sentidos, estos aprendizajes son falaces, pues no se asumen verdaderamente las implicaciones físicas y epistemológicas (la «visión del mundo») entrañadas por las nuevas ideas. Y en ningún caso se da aprendizaje actitudinal, ya que no se adopta la actitud de una mente científica abierta a las opiniones ajenas y a la revisión de las propias.

4) El aprendizaje significativo de los alumnos, conforme se desarrollan intelectualmente y acceden a conocimientos más complejos, parece darse por un proceso de cambio conceptual sucesivo en el que un marco intelectual es subsumido por otra visión del mundo. Esto refuerza los recientes estudios críticos de ciertos autores (Hewson y Beeth, 1995; Laburu, 1996) que matizan la consideración general del aprendizaje por cambio conceptual como una mera sustitución de unos marcos conceptuales por otros. En todo caso, todo ello debería contribuir a ensanchar en la mente del estudiante su visión de la ciencia y del universo.
HENNESSEY, M.G. (1991). Analysis of conceptual change and status change in sixth graders' concepts of force and motion. Madison (EEUU): Univ. of Wisconsin Press .

HEWSON, P.W. (1981). A conceptual change approach to learning science. European Journal of Science Education, 3, pp. 383-396.

HEWSON, P.W. (1982). A case study of conceptual change in special relativity: The influence of prior knowledge in learning. European Journal of Science Education, 4, pp. 61-78.

HEWSON, P.W. y BEETH, M.E. (1995). Enseñanza para un cambio conceptual: ejemplos de fuerza y movimiento. Enseñanza de las Ciencias, 13(1), pp. 25-35.

HEWSON, P.W. y THORLEY, N.R. (1989). The conditions of conceptual change in the classroom. International Journal of Science Education, 11, pp. 541-543. 


\section{INVESTIGACIÓN DIDÁCTICA}

LABURU, C.E. (1996). La crítica en la enseñanza de las ciencias: constructivismo y contradicción. Enseñanza de las Ciencias, 14(1), pp. 93-101.

MARLER, P. y TERRACE, H.S. (1984). The biology of learning. Nueva York: Springer.

MINSTRELL, J. (1982). Explaining the «at rest» condition of an object. Physics Teacher, 20, pp. 10-14.

MOSTERÍN, J. (1987). Conceptos y teorías en la ciencia. Madrid: Alianza Editorial.

OSBORNE, J.R. y FREYBERG, P. (1985). Learning in Science. The implications of children's Science. Londres: Heinemann.

PALINCSAR, A.S. y RANSOM, K. (1988). From the mystery spot to the thoughtful spot: The instructions of metacognitive strategies. The Reading Teacher, 41, pp. 784-789.

POSNER, G.J., STRIKE, K.A., HEWSON, P.W. y GERTZOG, W.A. (1982). Accommodation of a scientific conception: Towards a theory of conceptual change. Science Education, 66 , pp. 211-227.

REEVES, H. (1996). Últimas noticias del cosmos. Madrid: Alianza.

RESNICK, R. (1981): Introducción a la teoría especial de la relatividad. Méjico: Limusa.
SCOTT, P.H., ASOKO, H.M. y DRIVER, R.H. (1992). Teaching for a conceptual change: A review of strategies, en Duit, R., Goldberg, F. y Niedderer, H. (eds.). Research in Physics Learning: Theoretical Issues and Empirical Studies, pp. 310-329. (IPN: Kiel).

SINGH, J. (1982). Teorías de la cosmología moderna. Madrid: Alianza.

STRIKE, K.A. y POSNER, G.J., (1985). A conceptual change view of learning and understanding, en West \& Pines (eds.). Cognitive Structure and Conceptual Change. Orlando: Academic Press.

STRIKE, K.A. y POSNER, G.J. (1992). A revisionist theory of conceptual change, en Duschl \& Hamilton (eds.).Philosophy of science, cognitive psychology, and educational theory and practice. Nueva York: State University of New York Press.

THORLEY, N.R. (1990). The role of conceptual change model in the interpretation of classroom interactions. Madison: Univ. of Wisconsin Press.

VYGOTSKY, L.S. (1978). Mind in society. The development of higher psychological processes. Cambridge (EEUU): Harvard Univ. Press.

[Artículo recibido en septiembre de 1998 y aceptado en abril de 1999.] 